\title{
Short-term recognition memory for pitch: Effect of a priori probability on response times and error rates
}

\author{
MAX COLTHEART AND IAN CURTHOYS \\ MONASH UNIVERSITY, Clayton, Victoria, Australia
}

A previous finding (Bindra, Williams, \& Wise, 1965) that Ss respond "different" more quickly than "same" when comparing the pitch of two tones was confirmed. It was found that this effect could be enhanced by making same trials less frequent and that the effect could be reversed by making same trials more frequent. The experimental treatment also affected the error rates of the two responses. Error and latency results are discussed in terms of a model of $R T$ based upon a sequential-sampling and decision procedure, and are shown to be compatible with such a model. $A$ decision bias towards the response "different" must be taken into account when the model is applied. This bias appears to be influenced by the size of the interstimulus interval used.

Bindra, Williams, and Wise (1965) reported that when Ss were asked to decide as quickly as possible whether the second of two tones did or did not differ in pitch from the first tone, they took longer to respond "same" than to respond "different." Their Ss also gave more incorrect "different" responses than incorrect "same" responses.

One theoretical interpretation they proposed for these results was in terms of a simplified decision-theory model, which is illustrated by Fig. 1. They pointed out that if the decision criterion were located closer to the mean of the same distribution than to the mean of the different distribution, it would follow that the proportion of incorrect same responses, $p($ "S" $\mid$ D), would be less than the proportion of incorrect "different" responses, $p($ " $D$ " $\mid S)$, which was one of the two effects they observed.

They suggested further that response latency could be treated in this context by assuming that "latencies are maximal at the criterion point, the point of greatest uncertainty, and that they decrease progressively on both sides of it [Bindra et al, 1965, p. 1626]." It would follow from this that, if the criterion were nearer to the mean of the same distribution, the mean latency of correct same responses $\left(M_{S}\right)$ would exceed the mean latency of correct different responses $\left(M_{D}\right)$, which was the second of the effects they observed. It would also follow that this relationship would hold equally well for incorrect responses, although this is not discussed, and latency data for incorrect responses are not reported.

Consequently, the analysis they propose is consistent with the main features of their data if the decision criterion is assumed to be located asymmetrically. A question which arises immediately is, why should the criterion assume such a location? The optimal location of the criterion when the task is to choose between two Gaussian distributions of equal variance is jointly determined by the a priori probabilities of the two kinds of events and the costs and payoffs associated with the decision outcomes (the payoff matrix). When the a priori probabilities are equal and the payoff matrix is symmetric (or absent), the optimal location of the criterion is midway between the means of the two distributions. There is a possibility that the Ss in Bindra et al's experiment could not achieve this optimality because their task was not defined well enough. For instance, although the two kinds of trial (same and different) were equally frequent, it is not stated whether Ss were informed of this. If not, the fact that a priori probabilities were equal may not have had its customary effect. A minor aim of the present experiment was to elucidate this by emphasizing to Ss that a priori probabilities of the two kinds of trials were equal.

The main aim of this experiment, however, was to investigate the effects of manipulating the position of the criterion. If we denote by $P(S)$ the a priori probability of a trial on which the two tones have the same pitch, then, as $P(S)$ goes from 0.0 to 1.0 , the criterion will sweep from left to right across the distributions of Fig. 1. It is simple to see that some of the predicted effects of this are: (a) As $P(S)$ increases, $M_{S}$ will decrease and $M_{D}$ will increase. Therefore $\left(\mathrm{MS}_{\mathrm{S}}-\mathrm{MD}_{\mathrm{D}}\right)$ will be a decreasing monotonic function of $\mathrm{P}(\mathrm{S})$;

(b) As $\mathrm{P}(\mathrm{S})$ increases, the mean latency of incorrect "same" responses, $\bar{M}_{S}$, will decrease, and $\bar{M}_{D}$, the mean latency of incorrect "different" responses, will increase; hence $\left(\overline{\mathrm{M}}_{\mathrm{S}}-\overline{\mathrm{M}}_{\mathrm{D}}\right)$ will also be a decreasing monotonic function of $P(S)$;

(c) As $P(S)$ increases, the rate at which incorrect "same" responses occur will increase, and the rate at which incorrect "different" responses occur will decrease. Therefore the ratio of $P($ " $D$ " | S) to $P($ " $S$ " | D) will be a decreasing monotonic function of $P(S)$.

We therefore set out to replicate the results of Bindra et al, using $P(S)=0.50$ and emphasizing this to the $S$ s, and then for each $S$ running four further conditions involving other values of $P(S)$. The values of $P(S)$ used were $.10, .30, .50, .70$, and .90 .

Procedure

\section{METHOD}

Each of the four $\mathrm{Ss}^{1}$ served in five sessions, each session on a different day. A session consisted of 120 trials. The first 20 trials were a practice block. The remaining 100 trials consisted of four 25-trial blocks. There was a 2 -min rest period between blocks.

Each trial commenced with a 1-sec warning signal. This signal was a small light located close to the S's response buttons. One sec after the offset of this light, the first tone came on; it lasted for 4 sec. Next there was a $10-\sec$ interstimulus interval; then came the second tone, which also lasted for $4 \mathrm{sec}$. The next trial began $6 \mathrm{sec}$ after the offset of the second tone. Each tone was either $1050 \mathrm{~Hz}$ (denoted by $\mathrm{H}$ ) or else $1000 \mathrm{~Hz}$ (denoted by L). Thus there were four kinds of trials 2 : $\mathrm{HH}$ (first tone $1050 \mathrm{~Hz}$, second
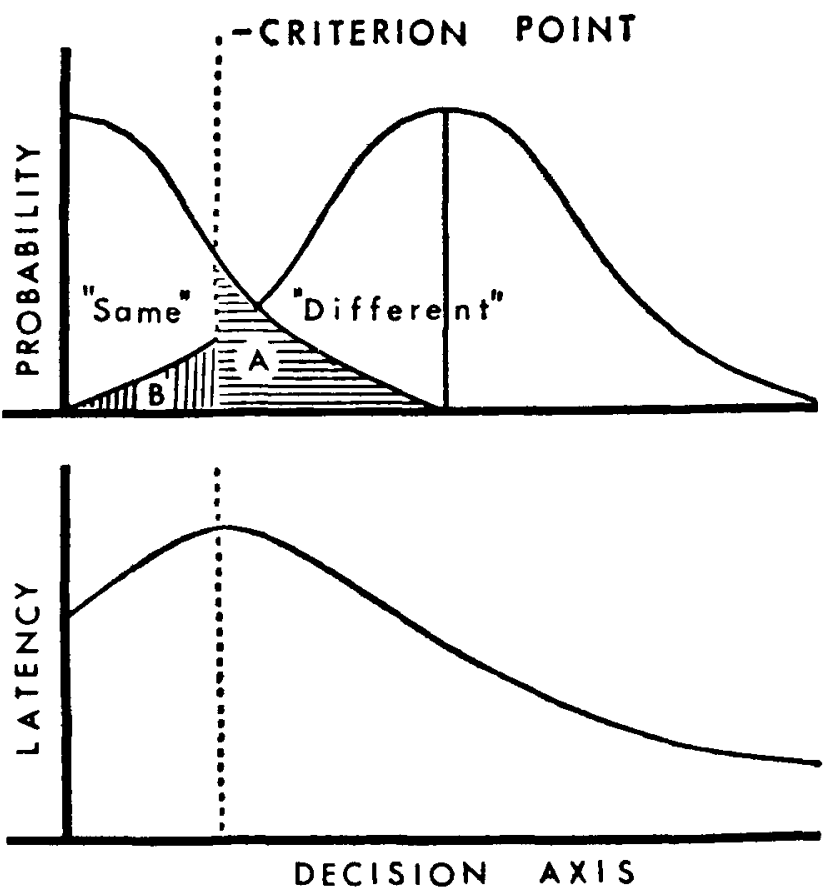

Fig. 1. The decision model proposed by Bindra et al (Fig. 1 from Bindra, Williams, \& Wise, 1965). 
tone $1050 \mathrm{~Hz}$ ), $\mathrm{Hl}, \mathrm{LH}$, and $\mathrm{LL}$. Within any session $\mathrm{HH}$ and $\mathrm{LL}$ trials were presented equally often, and $\mathrm{HL}$ and $\mathrm{LH}$ trials were presented equally often. However, $P(S)$, the proportion of trials on which the two tones had the same frequency (i.e., HH or LL trials), varied systematically from session to session. The values of $P(S)$ used were $.50, .70, .30, .10$, and .90 , and the sessions were given in this order to all four Ss.

Each $S$ was informed (before, and also after, the practice block of each session) of the value of $P(S)$ to be used during that session. For example, in the third session, each $S$ was told that although the sequence of trials was random, the paired tones of a trial would be the same on $30 \%$ of trials and different on the remaining $70 \%$, so that for any trial the probability that the second tone would be different from the first was. 70 . The $S$ was also told that the Es were interested in reaction time and that his task was to decide, as soon as possible after the second tone came on, whether it was the same as or different from the first tone, and to respond accordingly.

\section{Apparatus}

The $S$ sat in a modified dentist's chair in the center of an echo-deadened cubicle ( $10 \mathrm{ft} \times 8 \mathrm{ft} \times 8 \mathrm{ft}$ ) located in a larger laboratory. Attached to the right arm of the S's chair was a board on which the warning light and three (Rafi) press buttons were mounted. The $\mathrm{S}$ was instructed to rest his index finger on the center button except when responding. One of the outer buttons (the left button for two Ss, the right button for the other two) was labelled "SAME"; the other was labelled "DIFFERENT." The center of each response button was 1 in. from the center of the rest-button. The $S$ 's response consisted of moving his finger from the center button to the appropriate outer button and depressing this button.

In front of the $S$ was an 8 in. Rola Type C8MX loudspeaker, in a small infinite enclosure mounted on a microphone stand. The front of this enclosure was $3 \mathrm{ft}$ from the S's head. The SPL of each stimulus was $66 \mathrm{~dB}$ (measured at the position of the center of the S's head). The background noise level was approximately $59 \mathrm{~dB}$ SPL. These measurements were established and checked regularly with a Bruel and Kjaer Type 2203 sound level meter.

The stimuli were pure tones generated by a Bruel and Kjaer Type 1022 Beat Frequency Oscillator. Stimulus frequency was selected by the Frequency Increment control of this instrument. The onset and off set of the warning light and the two tones were controlled by a programmer, which was activated by pulses from two recycling Hunter Type $100 \mathrm{C}$ timers.

Reaction time was recorded by a Relion chronoscope which started simultaneously with the second tone on each trial and was stopped by the S's response. It was automatically reset during the interstimulus interval. The response keys were so wired that an erroneous response caused a red light to flash on the E's console. This console, the chronoscope, and the oscillator were located in the main laboratory, outside the S's cubicle. The programmer was located in another room; its output was fed to the E's control console by a 10 -core cable.

The apparatus is described in such detail because the omission of some of these details by Bindra et al (for instance, information about instructions to $S$, about the background noise SPL, and about the point at which the signal intensity was measured) made it difficult to relate their results to the results of other investigations.

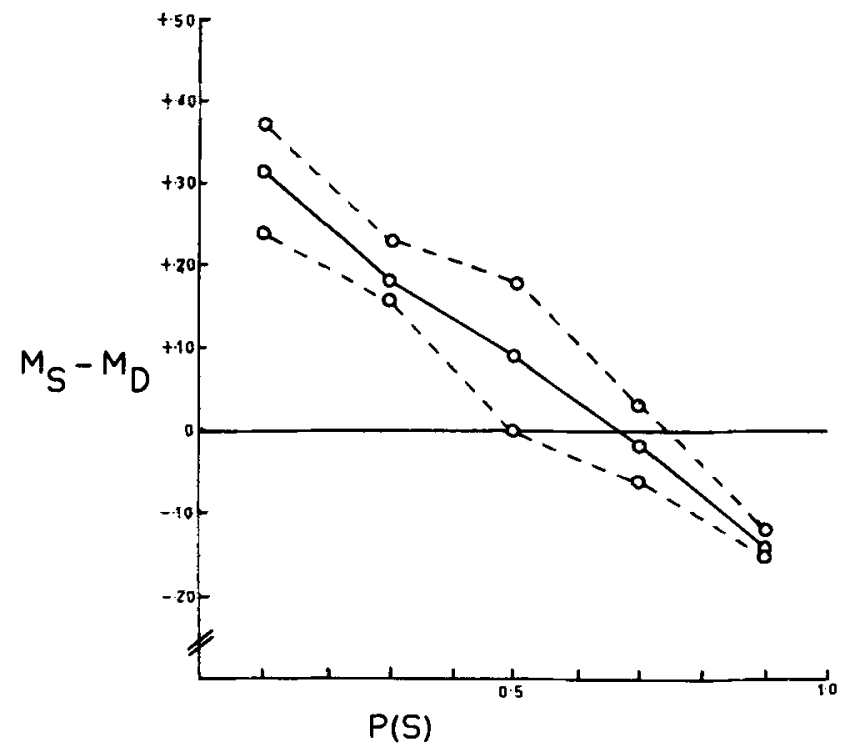

Fig. 2. Difference between $M_{S}$ (mean RT for correct "same" responses) and $M_{D}$ (mean RT for correct "different" responses) as a function of $P(S)$.

\section{RESULTS}

Relationship of $\left(\mathrm{M}_{S}-\mathrm{M}_{\mathrm{D}}\right)$ to $\mathrm{P}(\mathrm{S})$

Table 1 presents for each $S$ the value of $\left(\mathrm{MS}_{\mathrm{S}}-\mathrm{M}_{\mathrm{D}}\right)$ for each value of $\mathrm{P}(\mathrm{S})$. It will be seen that the prediction of a decreasing monotonic relationship between the two is supported reasonably well; the trend is uniform for all four Ss, except for reversals at $P(S)=.90$ for $S 4$ and $P(S)=.10$ for $S 1$. The solid curve of Fig. 2 shows the value (averaged over the four $S s)$ of $\left(M_{S}-M_{D}\right)$, for each value of $\mathrm{P}(\mathrm{S})$. This plot indicates a decreasing monotonic relationship between $\left(\mathrm{M}_{\mathrm{S}}-\mathrm{M}_{\mathrm{D}}\right)$ and $\mathrm{P}(\mathrm{S})$. Moreover, $\left(\mathrm{M}_{\mathrm{S}}-\mathrm{M}_{\mathrm{D}}\right)>0$ at $P(S)=0.50$, which supports the original finding of Bindra et al. This point is considered further below.

Table 2 presents the mean reaction times on $S$ trials and on $D$ trials for each $S$ at each value of $P(S)$.

\section{A Response Effect}

The upper dashed curve of Fig. 2 was obtained by averaging the data of $S 1$ and $S 2$, and the lower dashed curve by averaging the data of S3 and S4. For the former two Ss, t tests indicate that $\mathrm{M}_{\mathrm{S}}$ $>\mathrm{M}_{\mathrm{D}}$ at $\mathrm{P}(\mathrm{S})=0.50(\mathrm{t}=2.39, \mathrm{df}=97$, and $\mathrm{t}=3.27, \mathrm{df}=83$, respectively). For the latter two, the $t$ values obtained here are insignificant ( $t=.03$ and $t=.13$, respectively). It is unlikely, however, that this is because only two of our Ss behave like those of Bindra et al. The plot for S1 and S2 lies above the plot for the other two $S s$ at all five points, not just at $P(S)=0.50$. The reason for this becomes clear when we note that $S 1$ and $S 2$ were the Ss who had to move their index finger to the right to respond "same." Evidently, the movement to the right takes longer to execute than the movement to the left. This response effect enhances the Bindra effect for S1 and S2 and "masks" it for the other two Ss.

Two of our Ss spontaneously reported after the experiment that
Table 1

$\left(M_{S}-M_{D}\right)$ as a Function of $P(S)$ for Each Subject
Table 2

Mean RTs on S Trials and on D Trials as a Function of P(S) for Each Subject
$\left(M_{S}-M_{D}\right)$ in seconds

\begin{tabular}{lccccc}
$\mathrm{P}(\mathrm{S})$ & $\mathrm{S1}$ & $\mathrm{S} 2$ & $\mathrm{~S} 3$ & $\mathrm{~S} 4$ & Mean \\
\hline .10 & +.10 & +.63 & +.31 & +.16 & +.31 \\
.30 & +.16 & +.30 & +.23 & +.03 & +.18 \\
.50 & +.09 & +.26 & .00 & .00 & +.09 \\
.70 & -.01 & +.06 & -.05 & -.07 & -.02 \\
.90 & -.23 & -.01 & -.24 & -.05 & -.14 \\
\hline
\end{tabular}

\begin{tabular}{|c|c|c|c|c|c|c|c|c|}
\hline \multirow[b]{2}{*}{$P(S)$} & \multicolumn{2}{|c|}{ S] } & \multicolumn{2}{|c|}{$S 2$} & \multicolumn{2}{|c|}{ S3 } & \multicolumn{2}{|c|}{$\$ 4$} \\
\hline & $S$ & $D$ & $\mathrm{~S}$ & D & $S$ & D & $\mathrm{S}$ & D \\
\hline .10 & .95 & .85 & .85 & 1.48 & 0.83 & 0.52 & 0.58 & 0.42 \\
\hline .30 & 1.06 & .90 & 1.32 & 1.02 & 0.69 & 0.46 & 0.61 & 0.58 \\
\hline .50 & 1.06 & .97 & 1.42 & 1.16 & 0.85 & 0.85 & 0.69 & 0.69 \\
\hline .70 & 1.02 & 1.03 & 1.25 & 1.19 & 0.80 & 0.85 & 0.52 & 0.59 \\
\hline .90 & .80 & 1.03 & 0.82 & 0.83 & 0.80 & 1.04 & 0.49 & 0.54 \\
\hline
\end{tabular}




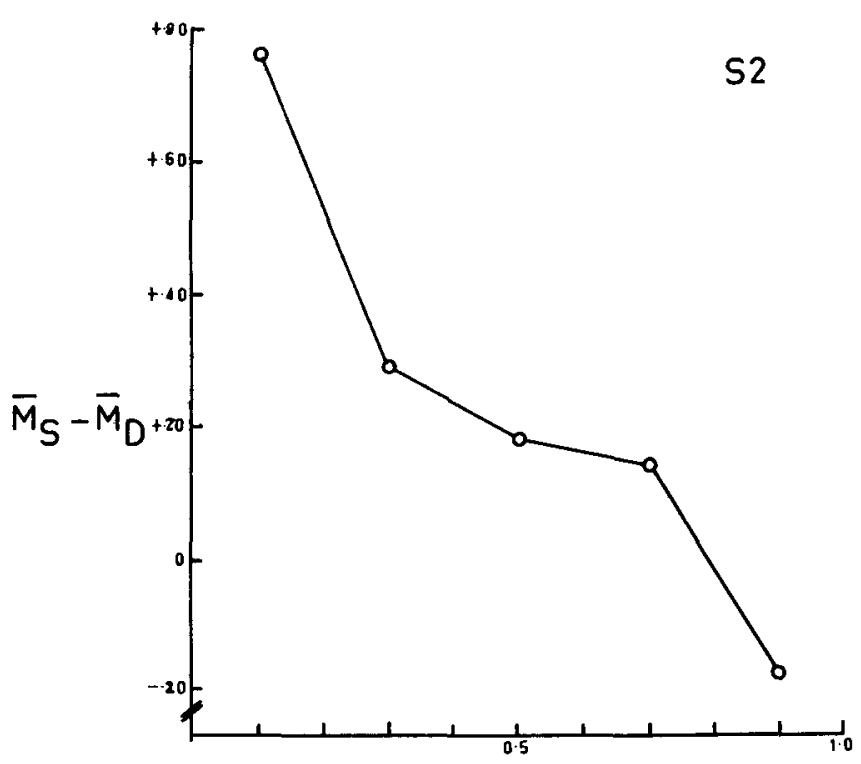

$P(S)$

Fig. 3. Difference between $M_{S}$ (mean RT for incorrect "same" responses) and $M_{S}$ (mean RT for incorrect "different" responses) as a function of P(S), for Subject 2.

they felt that they could move their index finger to the left more quickly and more easily than to the right. The other two Ss, when asked whether one response was easier to make than the other, also nominated the movement to the left as the easier response. It may be that the musculature of the index finger is responsible for this intrinsic difference in the speed and ease with which the two movements can be executed.

\section{Errors}

One feature of our data which differs from the data reported by Bindra et al, is that our Ss made many fewer errors than theirs. In their experiment, the average error rate was $11.7 \%$ with an interstimulus interval of $10 \mathrm{sec}$. Three of our Ss made very few errors (at $P(S)=0.50$, error rates of $1 \%, 1 \%$, and $0 \%$ were obtained); only $\mathrm{S} 4$ made errors at an appreciable rate (15\% at $\mathrm{P}(\mathrm{S})$ $=0.50$ ). Consequently, predictions involving errors can only be tested for this $\mathrm{S}$.

Figure 3 plots $\left(\overline{\mathrm{M}}_{\mathrm{S}}-\overline{\mathrm{M}}_{\mathrm{D}}\right)$ against $\mathrm{P}(\mathrm{S})$ for $\mathrm{S} 2$. This function has a monotonically declining trend, as predicted. Figure 4 plots the ratio of proportion of errors on $S$ trials to proportion of errors on $D$ trials. This ratio also tends to decrease monotonically with $P(S)$, as predicted.

\section{Decision Theory and Response Latency}

Although this experiment supported the model suggested by Bindra et al, it was far from a stringent test of this model; in fact, a variety of decision-theoretic approaches would make the same predictions. Some of these approaches are to be preferred to the model proposed by Bindra et al. One of the major deficiencies of the latter model is that it makes no provision for the inverse relationship between speed and accuracy in RT tasks. For instance, the decision axis referred to in Fig. 1 involves evidence collected on a trial for use in deciding which response to make. Since the $S$ responds before the offset of the stimulus, he does not collect as much evidence as he could. The longer he waits before responding, the more evidence he will collect; this will increase the probability of a correct response, but it will also increase the RT. Conversely, short RTs can only be achieved by collecting little

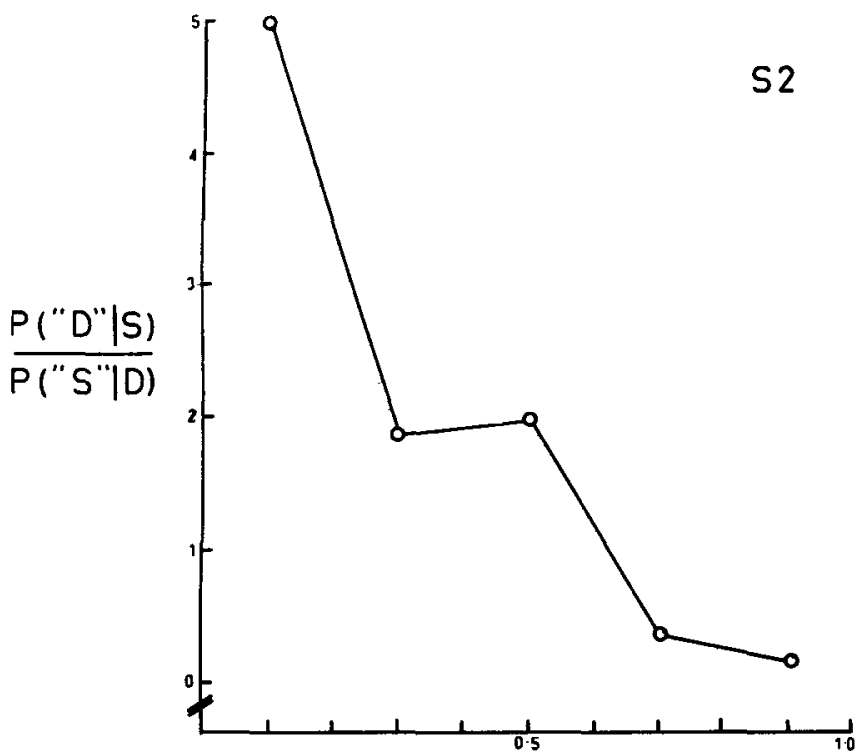

$P(S)$

Fig. 4. Ratio of $P($ “' $D$ " $\mid S)$, proportion of incorrect responses on $S$ trials, to $P($ " $S$ " | D), proportion of incorrect responses on $D$ trials, as a fuention of $\mathbf{P}(\mathbf{S})$, for Subject 2.

evidence before responding, and this decreases the probability of a correct response.

Considerations of this sort suggest that the $S$ ought to be viewed as sampling sequentially from the stimulus until he is able to make a decision concerning which response to make. Some recent discussions of RT have expressed similar ideas (e.g., Stone, 1960; Fitts, Peterson, \& Wolpe, 1963), and Swets and Birdsall (1967) have discussed the application of sequential analysis (Wald, 1947) in signal detection theory. These ideas can be applied to the "same"-"different" pitch discrimination task without difficulty, following Swets and Birdsall (1967, pp. 25-26).

The $S$ is supposed to commence sampling from the second tone as soon as it begins; we shall consider this sampling to be a discrete process, each sample being of very brief duration. The evidence yielded by sample $i$ is denoted by $e_{i}$. The likelihood ratio computed from sample $i, L_{i}$, is given by:

$$
L_{i}=\frac{P\left(e_{i} \mid \text { "same" stimulus }\right)}{P\left(e_{i} \mid \text { "different" stimulus }\right)}
$$

The values of $L_{i}$ obtained from the samples could be combined in various ways. Suppose this combination takes the form of multiplication. The $S$ bases his decision on the running product $L$, given by:

$$
\mathrm{L}=\mathrm{L}_{\mathrm{o}} \cdot \Pi \mathrm{L}_{\mathrm{i}}
$$

Here $\mathrm{L}_{\mathrm{o}}$ is the a priori odds of a "same" trial, i.e.,

$$
\mathrm{L}_{\mathrm{O}}=\frac{\mathrm{P}(\mathrm{S})}{\mathrm{P}(\mathrm{D})}
$$

There are two critical values of $\mathrm{L}_{S}$ and $\mathrm{L}_{\mathrm{D}}$. The S's decision rule is: if $L>L_{S}$, respond "same"; if $L<L_{D}$, respond "different"; if $\mathrm{L}_{\mathrm{S}}>\mathrm{L}>\mathrm{L}_{\mathrm{D}}$, draw another sample.

The duration of the decision-time component of RT will depend on the number of samples taken by $S$, since each sample contributes a finite amount of time to this component. The more samples taken before deciding which response is to be made, the longer RT will be. 3

The demands of speed and accuracy are coordinated by appropriate location of the criteria $\mathrm{L}_{S}$ and $\mathrm{L}_{\mathrm{D}}$. If increased speed 
is required, the criteria will be moved closer to $\mathrm{L}_{\mathrm{O}}$; now the expected sample size (and hence the average decision time) will decrease, but error rate will increase. Conversely, the criteria may be made more stringent by moving them away from $\mathrm{L}_{\mathrm{O}}$; accuracy will increase, but so will RT.

If $\mathrm{P}(\mathrm{S})>.50, \mathrm{~L}_{0}$ will be nearer to $\mathrm{L}_{S}$ than to $\mathrm{L}_{\mathrm{D}}$. In this case, $\mathrm{L}_{S}$ will be reached earlier than $\mathrm{L}_{\mathrm{D}}$, on the average; hence, $R T$ on same trials will shorter than RT on different trials. At the same time, $\mathrm{L}_{S}$ will be reached erroneously more of ten than $\mathrm{L}_{\mathrm{D}}$; hence there will be more incorrect "same" responses. The opposite effects will occur when $P(S)<.50$. This analysis predicts the effects on latencies and error rates observed when $P(S)$ was varied in the present experiment.

However, when $P(S)=P(D)$ and payoffs are symmetric (or absent), $L_{O}=1$ and $L_{S}$ and $L_{D}$ should be equidistant from $L_{O}$. Hence the two kinds of responses ought to have equal mean latencies and the two kinds of decision errors ought to be equally frequent. The fact that $\mathrm{M}_{\mathrm{S}}>\mathrm{M}_{\mathrm{D}}$, and that incorrect "different" responses predominate, at $P(S)=0.50$, indicates that on the average more samples are taken before the $S$ responds "same," i.e., he has a bias towards the decision "different."

Swets and Birdsall (1967) obtained evidence of a bias towards the response "Yes" in a Yes/No detection experiment in which the $S$ was allowed to defer responding and instead to repeat the trial at a small cost. They mentioned the results of Bindra et al, in this context. If one equates the presence of a stimulus in an absolute-threshold task and the presence of a difference between stimuli in a differential-threshold task, a bias towards "Yes" in the former task would correspond to a bias towards "Different" in the latter task.

This correspondence is an interesting one for a number of reasons. It emphasizes the fact that the experimental task used by Bindra et al, combines choice reaction time and signal detection. It also suggests that the measurement of RT in simple detection experiments might be of special interest. Furthermore, there is some evidence that these topics are complicated by an effect due to interstimulus interval.

\section{Effects of Interstimulus Interval}

Aiken and Lau (1966) investigated the relationship between interstimulus interval (ISI) and same-different pitch judgments. They found that as ISI increased, both incorrect and correct "different" responses occurred more often. This can be described as being the consequence of a shift upward of the two criteria; the degree of shift is related to the size of the ISI being used. Since this indicates an increase in the bias towards "different" as a function of ISI, it is predicted that $\left(\mathrm{M}_{\mathrm{S}}-\mathrm{M}_{\mathrm{D}}\right)$ would be an increasing function of ISI. Bindra et al, found that $\left(\mathrm{M}_{\mathrm{S}}-\mathrm{M}_{\mathrm{D}}\right)$ was $90 \mathrm{msec}$ at ISI $=1 \mathrm{sec}$ and $200 \mathrm{msec}$ at ISI $=10 \mathrm{sec}$; this difference was significant, and it is in agreement with the idea that the degree of bias towards the response "different" is positively related to the size of the ISI.

However, these data have numerous conflicting aspects. Firstly, reanalysis of the data obtained by Aiken and Lau indicates that there is a bias towards "same" when ISI $=.95 \mathrm{sec}$, since the proportion of correct "different" responses was .52 and the proportion of correct "same" responses was .75. This bias decreases and becomes a bias towards "different" for longer ISIs. At ISI $=1 \mathrm{sec}$ as we have seen, Bindra et al, found a bias towards "different."

Secondly, performance was poorer at an ISI of $10 \mathrm{sec}$ than at an ISI of 1 sec in Bindra's experiment, whereas there was no effect of ISI on percentage correct in Aiken and Lau's experiment. Wickelgren (1966), who filled the ISI with an interference tone, also reported that performance deteriorated as ISI increased.

Procedural differences doubtless are involved here. Aiken and Lau used frequency differences of $5 \mathrm{~Hz}$ or less; Bindra et al used $60 \mathrm{~Hz}$ differences, and thus gave their Ss a much easier task. Aiken and Lau used a constant direction of frequency difference within any block; Bindra et al, randomized the direction of difference. Since this meant that the first tone in Aiken and Lau's experiment was always $800 \mathrm{~Hz}$ and the second was either $800 \mathrm{~Hz}$ or else differed from $800 \mathrm{~Hz}$ by a fixed amount in a given direction, the task could have been performed to some extent even when the first tone was ignored. Konig (1957) presented Ss with a standard tone followed by 11 test tones, after each of which the $S$ had to respond "higher" or "lower" with reference to the initial standard tone. Performance in this task was nearly as good as that in a situation in which the standard tone preceded each test tone.

Keeping the first tone of the tone-pair constant over blocks seems undesirable in this kind of experiment; the task of deciding whether a second tone was higher or lower may be being performed by comparing it to a short-term memory trace of the first tone of that trial or by comparing it to a longer-term trace derived from previous trials in the block. Harris (1952, p. 97) has criticized the fixed standard procedure for this reason.

It is not clear, then, how discriminability changes as a function of ISI, nor how the criterion is affected. The data of Bindra et al and Wickelgren and Konig suggest that discriminability declines as ISI increases. The data of Aiken and Lau suggest that the two are unrelated. With ISIs of up to $15 \mathrm{sec}$, Harris (1952) found a very slight drop in discriminability as ISI increased, using a fixed standard, and a more marked decrease when a roving standard was used. Procedural differences may prevent these studies from being directly comparable. It seems likely that the bias towards "different" increases as a function of ISI, but the direction of this bias at very short ISIs is uncertain; moreover, the error rates with ISI = $1 \mathrm{sec}$ and ISI $=10 \mathrm{sec}$ in that data of Bindra et al do not clearly support this view of the relationship between ISI and the decision bias. 4

\section{Other Same-Different Tasks}

Bindra et al introduce their report with a general discussion of "same-different" judgments, and they appear to treat their data as generally applicable to this kind of judgmental task. However, subsequent work has indicated that their results were specific to some particular forms of the "same-different" task. Nickerson (1965) asked Ss to decide as quickly as possible whether the second of two visually presented English consonants was the same as the first or not. He found that the mean latency of the response "same" was less than the mean latency of the response "different." Sternberg (1966) found that when Ss were asked to decide whether a visually presented digit was the same as a single digit stored in short-term memory, the response "yes" had a lower mean latency than the response "no," i.e., "same" responses were faster than "different" responses.

Consequently, it cannot be maintained that the effect reported by Bindra et al is a characteristic of all "same-different" tasks.

\section{REFERENCES}

AlKEN, E. G., \& LAU, A. W. Memory for the pitch of a tone. Percept. \& Psychophys, 1966, 1, 231-233.

BINDRA, D., WILLIAMS, J. A., \& WISE, J. S. Judgments of sameness and difference: experiments on decision time. Science, 1965, 150,1625-1627.

EGETH $_{\text {; }}$ H. Parallel versus serial processes in multidimensional stimulus discrimination. Percept. \& Psychophys, 1966, 1, 245-252.

FITTS, P. M., PETERSON, J. R., \& WOLPE, G. Cognitive aspects of information-processing: II. Adjustments to stimulus redundancy. J. exp. Psychol., 1963, 65, 507-514.

GUILFORD, J. P. Psychometric methods New York: McGraw-Hill, 1954.

HARRIS, J, D. The decline of pitch discrimination with time. J. exp. Psychol, 1952, 43, 96-99.

KONIG, E. The effect of time on pitch discrimination thresholds under several psychophysical procedures. J. Acoust. Soc. Amer., 1957, 29, 606-612.

POSNER, M. I., \& KEELE, S. W. Decay of visual information from a single letter. Science, 1967, 158, 137-139.

RABBIT, P. M. A. Error correction times without external error signals. Nature, 1966, 21 2, 438.

ROSENBLITH, W. A., \& STEVENS, K. N. On the DL for frequency. $J$. Acoust. Soc. Amer. 1953, 25, 980-985.

STERNBLRG, S. High-speed scanning in human memory. Science, 1966, 153, 652-654.

STONE, M. Models for choice-reaction time. Psychometrika, 1960, 25, 251-260. 
SWETS, J. A., \& BIRDSALL, T. G. Deferred decision in human signal detection: a preliminary experiment. Percept. \& Psychophys, 1967, 2, 15-28.

WALD, A. Sequential analysis. New York: Wiley, 1947.

WICKELGREN, W. A. Consolidation and retroactive interference in shortterm recognition memory for pitch. J. exp. Psychol, 1966, 72, $250-259$.

\section{NOTES}

1. The Ss were three male members of the Department of Psychology and a female graduate. Two Ss were rejected due to undue variability and abnormally large RTs in initial sessions.

2. For each $S$ a two-way analysis of variance was carried out on the 500 RTs obtained (including both correct and incorrect responses). The two factors in the analysis were the nature of the first tone ( $\mathrm{H}$ vs $\mathrm{L}$ ) and the nature of the second tone ( $\mathrm{H}$ vs $\mathrm{L}$ ). The latter factor did not approach significance for any $S$, nor did the former factor for Subjects 2 and 4. No interaction effect was significant.

However, for S1, trials on which the first tone was high yielded significantly shorter RTs than trials on which the first tone was low. For S3 exactly the reverse effect occurred. In both cases the effect was consistent over all five experimental sessions. For S1, the mean RT on LL trials exceeded the mean RT on HH trials, and the mean RT on LH trials exceeded the mean RT on HL trials, for all five sessions. These inequalities were reversed for S3.

3. Rabbitt (1966) has studied an effect which is often noticed in RT experiments; the $S$ is able, immediately after making an incorrect response, to report that his response was incorrect. One could interpret this as indicating that Ss do not cease sampling from the stimulus immediately upon initiating a response. Information collected after response initiation would sometimes conflict with information collected earlier on and used in choosing a response. In this case the $S$ would know that his response was incorrect, although it would be too late to change the response.

4. The classical psychophysical time-order error may plausibly be described in terms of a change in the decision criterion as a function of the ISI. Guilford $(1954$, p. 309) reviews data indicating that there is a bias towards the judgment "greater" when the ISI is very short, and that this bias decreases to zero and reverses as ISI increases; it is argued above that a very similar effect is apparent in the data of Aiken and Lau (1966).

(Accepted for publication March 8, 1968.) 